\title{
Patient Perceptions and Effects of Single Community Pharmacy Systems: Key Elements for Transitions of Care
}

L. Amulya Murthy ${ }^{1}$, Klodiana Myftari ${ }^{2}$, Sheri Stensland ${ }^{3}$, Sonali G. Kshatriya ${ }^{4}$, Parisa Vatanka ${ }^{5}$, Susan R. Winkler ${ }^{6}$ and Spencer E. Harpe ${ }^{7}$

1. Undistributed Pharmacist, Jewel-Osco Pharmacy, Chicago, IL, USA

2. Ambulatory Care Pharmacist, Breakthrough Care Clinic, Wheaton, IL, USA

3. Associate Professor, Chicago, Midwestern University, Downers Grove, IL, USA

4. Staff Pharmacist, CVS Health Services, Inc. Wood Dale, IL, USA

5. Associate Director Corporate Alliances, American Pharmacists Association, Washington, DC, USA

6. Professor and Chair, Pharmacy Practice, Midwestern University, Downers Grove, IL, USA

7. Associate Professor of Pharmacy Practice, Midwestern University, Downers Grove, IL, USA

\begin{abstract}
The primary objective of the study was to evaluate baseline patient perception on single versus multiple community pharmacy systems. The secondary objective was to determine effects of a pharmacist provided educational intervention on perception of using a single community pharmacy system. This was a prospective survey based study implemented in a single grocery store chain pharmacy and one location of a large retail pharmacy. An anonymous pre-survey was administered to eligible patients. The primary investigator then delivered a brief educational intervention followed by an anonymous post survey. This aimed to determine if there was a change in perception of components assessed in the pre-survey. Data analysis was performed using descriptive statistics. Subjects reported using approximately 1.5 pharmacies. Major reasons included cost, location, and immunizations. $63 \%$ of subjects answered that their pharmacist will not have an accurate medication list when using multiple pharmacies, however, $92 \%$ of subjects responded that the pharmacist should be aware of all medications they take. Education on multiple pharmacy use may help protect against its risks. Cognizance of medications filled at other pharmacies and close communication between patients and pharmacists may help foster pharmacist-patient relationships while reducing the risk of multiple pharmacy use.
\end{abstract}

Key words: Multiple pharmacy use, transitions of care, medication reconciliation.

\section{Introduction}

Medication reconciliation is a key component in preventing medication errors and improving medication safety during transitions of care. The ASHP (American Society of Health System Pharmacists) and the APhA (American Pharmacists Association) define medication reconciliation as a thorough evaluation of medication use any time there is a change in drug therapy [1]. Medication reconciliation serves a

Corresponding author: L. Amulya Murthy, Pharm. D., Undistributed Pharmacist, Jewel-Osco Pharmacy, Chicago, IL, USA. fundamental role in MTM (Medication Therapy Management) services. The need for these services was prompted through the growth in medication use and increase in complex drug regimens [1, 2]. The MTM Service Model states that an essential component of these services is the "Personal Medication Record". Accordingly, ASHP and APhA emphasize the importance of patients keeping a personal medication record and stress that patients should share it with all of their healthcare providers [2].

The CDC (Centers for Disease Control and Prevention) reports that $21.7 \%$ of patients have had at 
least three and $10.6 \%$ of patients have had at least five prescriptions filled within the past thirty days [3]. A study recently published by Look and Mott investigated the prevalence of MPU (multiple pharmacy use) between 2003 and 2009. The study revealed that approximately $70 \%$ of subjects use two pharmacies. This remained constant while the number of subjects who use three or more pharmacies significantly increased from $24.1 \%$ to $29.1 \%$ with a $P$ value of $<0.001$ [4]. Interestingly, MPU occurred more frequently in insured, unemployed, Caucasian women of sixty-five years of age or older who had lower perceived health status, and multiple medical conditions. The most predominant reason for MPU noted in previous studies is driven by location and convenience to the patient's home, location of employment and healthcare professionals' office [4]. As patients continue to utilize multiple pharmacies it becomes challenging to maintain a comprehensive medication list. This results in medication discrepancies that are often preventable if medication records are contained within one location. Franic and colleagues investigated the criteria patients apply when selecting between community chains, independents, grocery stores, and discount store pharmacies. The study suggests location and price are factors involved in patients' selection of a pharmacy; however, the authors stated these factors were not determinants in all pharmacy settings [5]. Furthermore, the study found that patients' valued personnel qualities such as professionalism, friendliness, and caring across all pharmacy settings noted in the study [5].

Horn and colleagues conducted a survey-based study through the ISMP (Institute of Safe Medication Practices) to evaluate the awareness of medication reconciliation in the community pharmacy setting and the degree of communication between those community pharmacies and emergency departments [6]. The basis of the study was that the success of medication reconciliation in an institutional setting depends on communication of the most up-to-date medication record from the outpatient venue. The study revealed that many patients do not provide a comprehensive medication list at their medical visits and this presents one of the largest barriers to medication reconciliation in the emergency department. Additionally, the study indicated that when patients use multiple pharmacies it becomes problematic for emergency departments to obtain and validate medication information [6].

The state of Oregon recently enacted a policy to fine pharmacies or even revoke licenses for providing coupons and other incentives to encourage patients to transfer medications between pharmacies that do not share a common database. The state board surveyed pharmacists in Oregon who expressed concern that patients were receiving prescriptions from multiple pharmacies and this was putting the pharmacist at risk for missing critical drug interactions [7]. It is important for pharmacists to educate patients that although they may be acquiring coupons to use for future expenditures, the cost of reconciling a medication error from pharmacists' inability to review a complete medication record is estimated to be around $\$ 9,000$ or more depending on the outcome [8]. Studies have demonstrated why patients use multiple pharmacies, and movements like the prescription transfer prohibition in Oregon have indicated how pharmacists perceive multiple pharmacy use. However, a key element lacking in the literature is the patient perception on the use of a single versus multiple community pharmacy system.

The primary goal of this study is to assess patient perception on the use of single versus multiple community pharmacy systems at baseline. The secondary objective is to determine the impact of an educational intervention on patient perception of single community pharmacy system use.

\section{Methods}

The pre-/post- study began in November 2013 upon receiving IRB approval. Subjects had to be eighteen 
years or older, able to read and communicate in English and utilized Dominick's Pharmacy site \#1995, Walgreens site \#9638, or Walgreens site \#6518. Subjects were invited to participate in the study by the primary investigator if they utilized the aforementioned pharmacies to fill prescription or utilize other services.

It was made known that participation in the study was completely voluntary and the purpose, study methods, and participants' rights were described to the subject in an informational handout. Consenting subjects received a pre-survey from the primary investigator, which was designed to obtain demographic data including age, gender, yearly income, type of insurance coverage, number of prescription medications used in the past six months, number of different pharmacies subjects have used within the past year, which pharmacies subjects have used, why they use multiple pharmacies, and whether or not the subject keeps a medication list. Additionally, the pre-survey was designed to assess the subject perception on the use of single versus multiple community pharmacy systems. Upon completion of the pre-survey, the subject's survey was placed into anonymous folder.

Next, the primary investigator provided the subject with a brief educational handout. The focus of this five-minute educational intervention highlighted the risks associated with multiple community pharmacy use such as pharmacists' ability to screen for drug-drug interactions, reduction of medication errors during transitions of care, and avoidance of therapy duplication on medication records. The educational component reinforced the pharmacist-patient relationship by explaining the roles and responsibilities of a community pharmacist. Following the intervention, a post-survey was administered to subjects that mirrored the pre-survey to determine if their perception on the use of single versus multiple community pharmacy systems changed. Upon completing the post-survey subjects placed the survey into the anonymous folder previously mentioned. The pre and post intervention surveys were coded utilizing a random number with no significance or identity to the subject. The number on the survey will was used for matching the pre-survey with the immediate post-survey. Data from the study was compiled and analyzed using descriptive statistics.

\section{Results}

Twenty-five surveys were completed from this data demographic information was omitted on four surveys. One survey contained demographic information, but not patient perception data, and thus it was felt appropriate to exclude those surveys from analysis. The ratio of males and females was approximately the same with $52 \%$ being female. On average subjects claimed to fill about four prescriptions a month while utilizing 1.5 pharmacies. The mean of 1.5 resulted as most subjects stated to use one to two pharmacies. $71 \%$ of subjects in the study had private insurance, while $19 \%$ and $10 \%$ had Medicare/Medicaid or a combination of third party plans, respectively.

Reason for multiple pharmacy use was predominantly driven by cost. Location and immunizations were also contributing factors for MPU as depicted in Figure. 1.

The final component of the pre-survey inquired about whether or not subjects kept a medication list. It was found that eleven subjects keep a medication list. Eight of these subjects reported creating the medication list themselves, four subjects stated they received their medication list from their doctor's office, and one subject noted that they received their medication list from their pharmacy. This portion of the survey was checked all that apply, and may have resulted in subjects receiving medication lists from multiple sources. Furthermore, when asked how often the subject updates their medication list $52 \%$ of subjects update their medication list upon initiation of a new medication. $33 \%$ of subjects revise their medication list anytime a dose changes or a drug is discontinued, and only $10 \%$ of subjects update their medication list every six months. Table 2 describes these trends. 


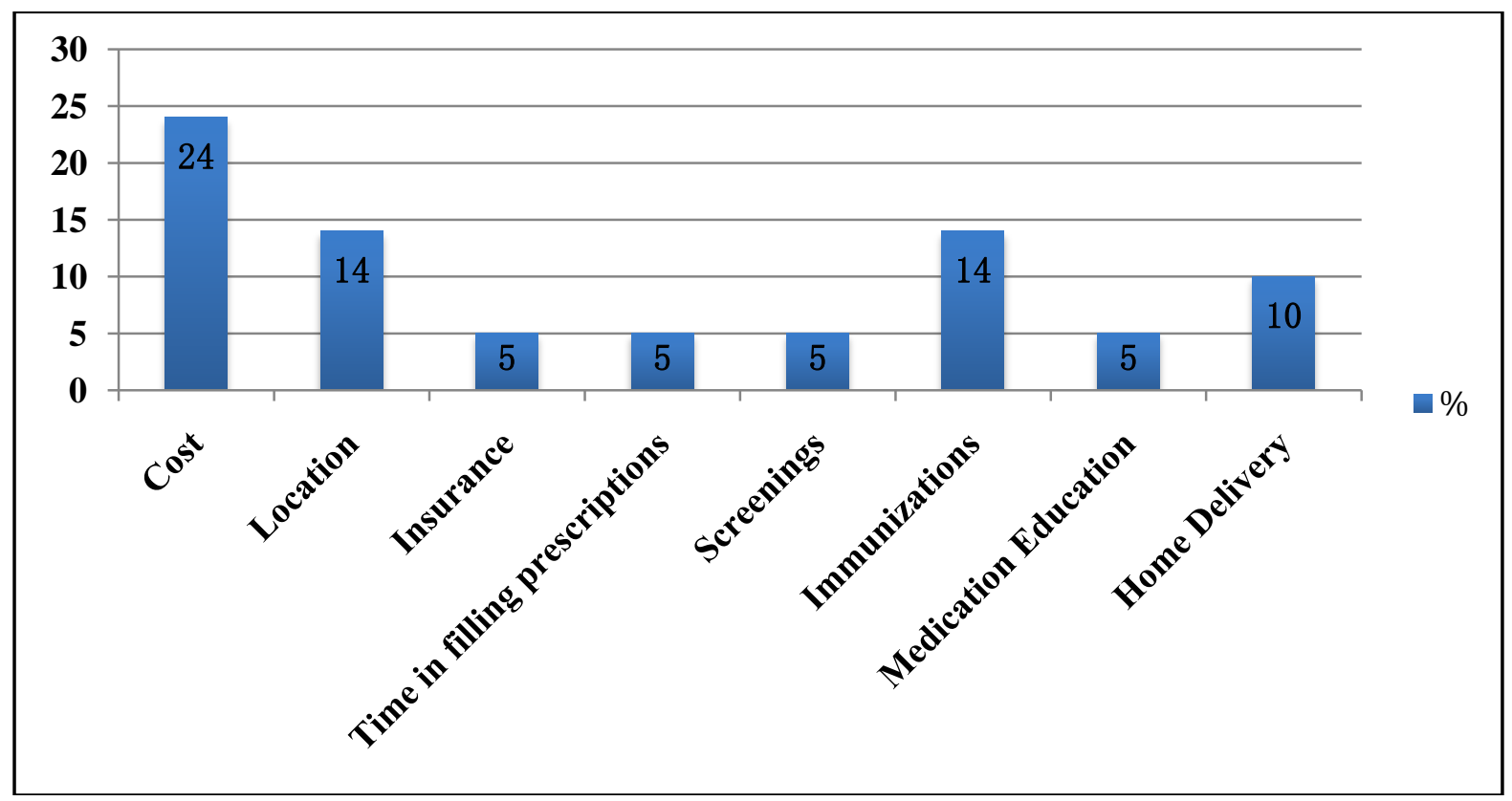

Fig. 1 Reasons for multiple pharmacy use.

Table 2 Where patients obtain their medication list.

\begin{tabular}{ll}
\hline Subject creates it themselves & $28 \%(8)$ \\
\hline Doctor's Office & $19 \%(4)$ \\
Pharmacy & $5 \%(1)$ \\
\hline
\end{tabular}

Table 3 How often subjects update their medication list.

\begin{tabular}{ll}
\hline Initiation of a new medication & $52 \%(11)$ \\
\hline Change in dose & $33 \%(7)$ \\
Medication discontinuation & $33 \%(7)$ \\
Every six months & $10 \%(2)$ \\
\hline
\end{tabular}

Next, the pre-survey assessed subject perception of multiple pharmacy use using a Likert scale. The first four questions focused on whether or not subjects agree or disagree with the potential health risks of MPU, and the remaining questions sought to assess subjects' perception of the role of a community pharmacist. Interestingly, $63 \%$ of subjects agreed or strongly agreed that the pharmacist would not have an accurate medication list, $71 \%$ believed screening for drug-drug interactions becomes compromised, $67 \%$ perceived medication duplications will occur, and 79\% recognized there are health risks with multiple pharmacy use. Assessing the subjects' perception on the role of a community pharmacist, $92 \%$ and $96 \%$ answered they view a pharmacist as a part of their healthcare team and the pharmacist should be aware of all the medications they take, respectively.

The next portion of the survey inquired about patient preference of services offered in the pharmacy. $100 \%$ of subjects ranked medication education as a priority. OTC (over-the-counter) triage and private consultation were ranked second and third at $96 \%$ and $88 \%$, respectively.

\section{Discussion}

This study observed somewhat similar results to previous studies in the reasons subjects utilize multiple pharmacies. Location was a major driving factor, and our study found that cost and immunizations were additional indications for multiple pharmacy use. 
Examining the patient perception questions $63 \%$ of subjects agreed or strongly agreed that the pharmacist would not have an accurate medication list when using multiple pharmacies; however, $96 \%$ of subjects responded that their pharmacist should be aware of all the medications they take. This suggests a discrepancy between what the subject knows about multiple pharmacy use and what they expect from their pharmacist.

Furthermore, only 5\% of subjects received their medication list from a pharmacy. This may offer a potential valuable service the pharmacy can provide in lieu of multiple pharmacy use. As previously mentioned, subjects indicated they valued medication education, over-the-counter triage, and private consultation services. Expansion of these services may encourage single pharmacy use as well as foster pharmacist-patient relationships.

This study was conducted in three locations, one grocery store pharmacy and two large retail chain pharmacies. The first location closed for business shortly after data collection began. As a result subjects were concerned about the status of their medications and medication records and were not interested in completing the post-survey. Surveys that were completed at the second large retail chain had a total of seven completed post-surveys. Since about only one-third of post-surveys were completed it was felt appropriate to be excluded from the final data analysis.

Limitations of the study include the small sample size, and short duration of data collection. Due to the small sample size it is undetermined whether or not factors such as third party status and yearly income influence MPU. Most of the data was collected at Dominick's Pharmacy Site \#1995 where several patients had a strong pharmacist-patient relationship. This may have hindered true subject responses regarding multiple pharmacy use.

\section{Conclusion}

This study provides a meaningful pharmacist-patient conversation about multiple pharmacy use and its associated harms. Some patients may insist on utilizing multiple pharmacies due to cost and third party purposes. In such instances, the pharmacist may offer services such as creating medication lists and documenting additional medications the patient is taking to properly screen for drug-drug interactions. Although $92 \%$ of subjects in the study stated that they view a pharmacist as a part of their healthcare team there is still substantial growth for professional advocacy to allow patients to see pharmacists as equally competent healthcare providers.

\section{References}

[1] Improving Care Transitions. 2012. "Optimizing Medication Reconciliation.” J. Am. Pharm. Assoc. 52: e43-e52.

[2] American Pharmacists Association and National Association of Chain Drug Stores Foundation. 2008. "Medication Therapy Management in Community Pharmacy Practice: Core Elements of an MTM Service (Version 2.0)." J. Am. Pharm. Assoc. 48: 341-53.

[3] Centers for Disease Control and Prevention. 2013. "Therapeutic Drug Use." U.S. Department of Health and Human Services. Accessed September 6, 2013.

[4] http://www.cdc.gov.

[5] Look, K. A., and Mott, D. A. 2013. "Multiple Pharmacy Use and Types of Pharmacies Used to Obtain Prescriptions." J. Am. Pharm. Assoc. 53: 601-10.

[6] Franic, D. M., Haddock, S. M., Tucker, L. T., and Wooten, N. 2008. "Pharmacy Patronage: Identifying Key Factors in the Decision Making Process Using the Determinant Attribute Approach." J. Am. Pharm. Assoc. 48 (1): 71-85.

[7] Horn, D., Gaunt, M. J., and Vaida, A. J. 2013. "Institute for Safe Medication Practices.” Medication Reconciliation: A Survey of Community Pharmacies and Emergency Departments. Accessed September 6, 2013.

[8] http://www.psqh.com.

[9] Gunderson, L. 2013. "Promotions end for Consumers who Transfer Prescriptions." The Oregonian. Accessed September 6, 2013.

[10] http://www.oreonlive.com.

[11] Jamaldinian, M. 2013. "Services for Pharmaceutical Consumer." Health Information Corporation. Accessed September 6, 2013.

[12] http://www.consumer-health.com/services. 